\title{
Predicting Thromboembolic and Bleeding Event Risk in Patients with Non-Valvular Atrial Fibrillation: A Systematic Review
}

\author{
Ethan D. Borre ${ }^{1}$ Adam Goode ${ }^{1,2}$ Giselle Raitz ${ }^{1}$ Bimal Shah ${ }^{1,3}$ Angela Lowenstern ${ }^{4,5}$ \\ Ranee Chatterjee $^{1}$ Lauren Sharan $^{1}$ Nancy M. Allen LaPointe ${ }^{1,6}$ Roshini Yapa $^{7}$ J. Kelly Davis ${ }^{8}$ \\ Kathryn Lallinger ${ }^{1,4,9}$ Robyn Schmidt ${ }^{1,4,9}$ Andrzej Kosinski ${ }^{10}$ Sana M. Al-Khatib ${ }^{4,5}$ \\ Gillian D. Sanders ${ }^{1,4,8,9}$
}

\footnotetext{
${ }^{1}$ Department of Medicine, Duke University School of Medicine, Durham, North Carolina, United States

${ }^{2}$ Department of Orthopedic Surgery, Duke University School of Medicine, Durham, North Carolina, United States

${ }^{3}$ Livongo, Mountain View, California, United States

${ }^{4}$ Duke Clinical Research Institute, Duke University School of

Medicine, Durham, North Carolina, United States

${ }^{5}$ Division of Cardiology, Duke University School of Medicine,

Durham, North Carolina

6 Premier Inc., Charlotte, North Carolina, United States

${ }^{7}$ Department of Medicine, University of Colorado, Aurora, Colorado, United States

${ }^{8}$ Duke-Margolis Center for Health Policy, Duke University, Durham, North Carolina, United States

${ }^{9}$ Evidence-Based Practice Center, Duke Clinical Research Institute, Duke University School of Medicine, Durham, North Carolina, United States

10 Department of Biostatistics and Bioinformatics, Duke University School of Medicine, Durham, North Carolina, United States
}

\author{
Address for correspondence Gillian D. Sanders, PhD, Duke Clinical \\ Research Institute, Duke Box 3485, 7020 North Pavilion Building, \\ Durham, NC 27710, United States (e-mail: gillian.sanders@duke.edu).
}

Thromb Haemost 2018;118:2171-2187.

\begin{abstract}
Keywords

- non-valvular atrial fibrillation

- stroke risk

- bleeding risk
\end{abstract}

Background Atrial fibrillation (AF) is a common cardiac arrhythmia that increases the risk of stroke. Medical therapy for decreasing stroke risk involves anticoagulation, which may increase bleeding risk for certain patients. In determining the optimal therapy for stroke prevention for patients with AF, clinicians use tools with various clinical, imaging and patient characteristics to weigh stroke risk against therapyassociated bleeding risk.

Aim This article reviews published literature and summarizes available risk stratification tools for stroke and bleeding prediction in patients with AF.

Methods We searched for English-language studies in PubMed, Embase and the Cochrane Database of Systematic Reviews published between 1 January 2000 and 14 February 2018. Two reviewers screened citations for studies that examined tools for predicting thromboembolic and bleeding risks in patients with AF. Data regarding study design, patient characteristics, interventions, outcomes, quality, and applicability were extracted. received

September 10, 2018

accepted after revision

September 27, 2018 (c) 2018 Georg Thieme Verlag KC Stuttgart · New York
DOI https://doi.org/ $10.1055 / \mathrm{s}-0038-1675400$. ISSN 0340-6245. 
Results Sixty-one studies were relevant to predicting thromboembolic risk and 38 to predicting bleeding risk. Data suggest that $\mathrm{CHADS}_{2}, \mathrm{CHA}_{2} \mathrm{DS} \mathrm{S}_{2}-\mathrm{VASC}$ and the age, biomarkers, and clinical history $(\mathrm{ABC})$ risk scores have the best evidence for predicting thromboembolic risk (moderate strength of evidence for limited prediction ability of each score) and that HAS-BLED has the best evidence for predicting bleeding risk (moderate strength of evidence).

Limitations Studies were heterogeneous in methodology and populations of interest, setting, interventions and outcomes analysed.

Conclusion $\mathrm{CHADS}_{2}, \mathrm{CHA}_{2} \mathrm{DS}_{2}-\mathrm{VASC}$ and $\mathrm{ABC}$ scores have the best prediction for stroke events, and HAS-BLED provides the best prediction for bleeding risk. Future studies should define the role of imaging tools and biomarkers in enhancing the accuracy of risk prediction tools.

Primary Funding Source Patient-Centered Outcomes Research Institute (PROSPERO \#CRD42017069999)

\section{Introduction}

Atrial fibrillation (AF) is the most common cardiac arrhythmia seen in clinical practice, occurring in up to 6.1 million people in the United States and accounting for approximately one-third of hospitalizations for cardiac rhythm disturbances. ${ }^{1-3}$ Further, AF is associated with significant morbidity and mortality, including increased risk of embolic stroke, heart failure and cognitive impairment; reduced quality of life; and higher overall mortality. ${ }^{4-6}$

Optimal clinical management of AF is critical to reducing this associated morbidity and mortality, and includes prevention of AF-related thromboembolic events in at-risk patients. Vitamin K antagonists or direct oral anticoagulants have been shown to reduce thromboembolic events, but long-term use of these medications puts certain patients at higher risk for serious bleeding events. As such, accurate risk stratification for both thromboembolic and bleeding risk is paramount in identifying patients for whom anti-thrombotic therapy would achieve maximum treatment benefit with the lowest risk of complications.

Unfortunately, it is challenging to estimate the trade-off between stroke risk and risk of bleeding complications from long-term anticoagulation therapy because many risk factors for stroke are also associated with increased risk of bleeding. There are several available risk stratification tools used to determine thromboembolic and bleeding risk that incorporate diagnostic imaging as well as patient factors such as age, sex and history of heart disease to aid in clinical decisionmaking around treatment strategies for AF. Of the many available risk stratification tools, the 2014 American Heart Association/American College of Cardiology/Heart Rhythm Society (AHA/ACC/HRS) guideline for patients with AF recommends the use of the $\mathrm{CHA}_{2} \mathrm{DS}_{2}$-VASc score to estimate the stroke risk and the HAS-BLED score for bleeding risk. ${ }^{7-9}$ However, these risk scores have been previously categorized as poor to moderate predictors of risk, and are just two of many different published and validated methods for assessing stroke and bleeding risk in patients with AF. Because patients, providers and policymakers have numerous decision tools that could inform treatment decisions and policy recommendations, there is a need for a compilation and analysis of the currently available data. This systematic review was commissioned by the Patient-Centered Outcomes Research Institute (PCORI) to update a 2013 Agency for Healthcare Research and Quality (AHRQ) review, ${ }^{10}$ with a focus on evaluating the comparative diagnostic accuracy and impact on clinical decision-making of available clinical and imaging tools and associated risk factors for predicting thromboembolic and bleeding risk in U.S. patients with AF. Our findings related to stroke prevention treatments are discussed in a companion paper.

\section{Methods}

Methods for this updated comparative effectiveness review (CER) follow the AHRQ's Methods Guide for Effectiveness and Comparative Effectiveness Reviews (hereafter referred to as the Methods Guide $)^{11}$ and Methods Guide for Medical Test Reviews (hereafter referred to as the Medical Test Guide). ${ }^{12}$ This article is part of the larger updated review; complete details of our methods, including exact search strings, and full results and conclusions can be found in the full report, available at www.effectivehealthcare.ahrq.gov.

\section{Defining the Key Questions}

PCORI convened two multi-stakeholder virtual workshops in December 2016 and January 2017 to (1) gather input from end users and clinical, content and methodological experts on scoping for the updated review; (2) prioritize the key questions; (3) discuss changes in the evidence base since the 2013 review; and (4) explore emerging issues in AF. The protocol for this systematic review was informed by discussion at the January 2017 workshop and builds upon the original report. The final protocol for this review is posted on the Effective Health Care (EHC) website (www. effectivehealthcare.ahrq.gov) and registered at PROSPERO (CRD42017069999). 
In this article, we summarize the evidence and findings related to two key questions (KQs): (1) In patients with nonvalvular $\mathrm{AF}$, what are the comparative diagnostic accuracy and impact on clinical decision-making (diagnostic thinking, therapeutic and patient outcome efficacy) of available clinical and imaging tools and associated risk factors for predicting thromboembolic risk? and (2) In patients with nonvalvular AF, what are the comparative diagnostic accuracy and impact on clinical decision-making (diagnostic thinking, therapeutic and patient outcome efficacy) of clinical tools and associated risk factors for predicting bleeding events?

\section{Data Sources and Study Selection}

In consultation with an expert medical librarian, we searched PubMed, Embase and the Cochrane Database of Systematic Reviews for relevant literature published from 1 August 2011 to 14 February 2018 (exact search strings are given in - Supplementary Table \$1, available in the online version). We supplemented electronic searches with a manual search of citations from a set of systematic review articles. Our findings were combined with those from the 2013 review, and so the literature summarized here reflects evidence back through 1 January $2000 .{ }^{10}$ Due to updates in inclusion criteria, any studies excluded from the original review were also re-reviewed for eligibility. We used search criteria to identify relevant on-going clinical trials through ClinicalTrials.gov as well as citations to guide the conclusions (-Supplementary Table S1, available in the online version).

Our pre-specified inclusion and exclusion criteria are given in - Supplementary Table S2 (available in the online version). We included English-language studies of adults with non-valvular AF (including atrial flutter) that reported the efficacy of clinical or imaging tools, or patient risk factors, on predicting thromboembolic and/or bleeding outcomes. Clinical or imaging tools considered for predicting thromboembolic events were $\mathrm{CHADS}_{2}$ score, $\mathrm{CHA}_{2} \mathrm{DS}_{2}$-VASc score, Framingham risk score, age, biomarkers, and clinical history (ABC) stroke score, transthoracic and transoesophageal echocardiography, computed tomography scans and cardiac magnetic resonance imagings (MRIs). Clinical or imaging tools considered for predicting bleeding events were the HAS-BLED score, HEMORR ${ }_{2}$ HAGES score, Anticoagulation and Risk Factors in Atrial Fibrillation (ATRIA) score, Bleeding Risk Index (BRI) and ABC bleeding risk score. Thromboembolic outcomes included cerebrovascular infarction, transient ischaemic attack and systemic embolism (excluding pulmonary embolism and deep vein thrombosis). Bleeding outcomes included haemorrhagic stroke, intra-cranial haemorrhage (ICH) and major and minor bleeds. We excluded studies that evaluated patients exclusively from Asia, Africa or the Middle East. We also sought to identify studies which used the same patients and linked these as companion papers to an individual study.

Data Extraction and Quality Assessment of Individual Studies

Pairs of investigators screened all citations and abstracts for eligibility, and those considered relevant by either investi- gator advanced to full-text review. Paired investigators then reviewed all full-text articles and resolved disagreements through discussion or adjudication by a third investigator. Paired investigators independently abstracted data and assessed study quality. Disagreements were resolved by consensus or arbitration by a third investigator. Articles that represented evidence from the same overall study were linked to avoid duplication of patient cohorts.

We assessed methodological quality, or risk of bias, for each individual study using tools specific to the study's characteristics. For studies assessing diagnostic accuracy, we used the Quality Assessment of Diagnostic Accuracy Studies-2 (QUADAS-2) tool. ${ }^{13}$ Our outcome-specific quality assessment classified study outcomes as containing low, medium or high risk of bias as defined by QUADAS- 2 .

\section{Data Synthesis and Analysis}

We summarized key features of the included studies for each $\mathrm{KQ}$ including information on study design; patient characteristics; clinical settings; diagnostic tools; and intermediate, final and adverse event outcomes. We ordered our findings by diagnostic comparison, and then within these comparisons by outcome, with long-term final outcomes emphasized.

Grouping interventions by prediction tool, we determined the feasibility of completing a quantitative synthesis (i.e. meta-analysis) based on the volume of relevant literature (at least three appropriate studies), conceptual homogeneity of the studies in terms of study population and outcomes and completeness of the reporting of results. When at least three comparable studies reported the same outcome, we used the R statistical package (version 3.1.2) (The R Foundation) with the 'metafor' meta-analysis library (version 1.9-7) to synthesize available $c$-statistics, which quantify the discrimination ability of the studied tools, for each appropriate thromboembolic or bleeding risk prediction tool. We used the random-effects DerSimonian and Laird estimator ${ }^{14}$ to generate summary values. In addition, we used the Knapp-Hartung approach to adjust the standard errors of the estimated coefficients. Since the diagnostic tools considered are not binary, it was not possible to consider summary receiver operating characteristic curves. When possible, the $c$-statistics were pooled by considering their estimated values (point estimates) and confidence intervals (CIs), and the 'generic point estimates' effect specification option in the Comprehensive Meta-Analysis software. For a clinical prediction rule, we assumed that a $c$ statistic of $<0.6$ had no clinical value, 0.6 to 0.7 had limited value, 0.7 to 0.8 had modest value and $>0.8$ had discrimination adequate for genuine clinical utility. ${ }^{15}$

\section{Strength of Evidence}

We assigned strength of evidence scores for each diagnostic tool using the approach described in the AHRQ's Methods Guide. ${ }^{11,16}$ We assessed five domains: study limitations; consistency; directness; precision; and reporting bias, which includes publication bias, outcome reporting and analysis reporting bias. These domains were considered qualitatively, 
and a summary rating of high, moderate or low strength of evidence was assigned for each outcome after independent assessment and discussion by two reviewers. In cases where ratings were impossible or imprudent to make, a grade of 'insufficient' was assigned.

\section{Role of the Funding Source}

This topic was nominated and funded by PCORI for systematic review by an Evidence-based Practice Center in partnership with AHRQ. A representative from AHRQ served as a Contracting Officer's Representative (COR) and provided technical assistance during the conduct of the full evidence report. The AHRQ COR and PCORI program officers provided comments on draft versions of the protocol and full evidence report. PCORI and AHRQ did not directly participate in the literature search; determination of study eligibility criteria; data analysis or interpretation; or preparation, review or approval of the manuscript for publication.

\section{Results}

We screened 11,274 publications and found 45 articles (25 studies) for KQ1 and 34 articles (18 studies) for KQ2 that investigated our included tools for determining stroke or bleeding risk in patients with non-valvular AF and that met the other inclusion criteria. We combined these newly identified studies with those included in the 2013 review, yielding a total of 83 articles (61 studies) for KQ1 and 57 articles (38 studies) for KQ2 included in this updated review (-Fig. 1). Complete results of the review, including longterm stroke and bleeding risk summaries, are in the full report.

\section{Predicting Thromboembolic Risk in Patients with AF}

We considered findings from the 61 studies reporting the predictive value of the $\mathrm{CHADS}_{2}, \mathrm{CHA}_{2} \mathrm{DS}_{2}$-VASc, Framingham and $\mathrm{ABC}$ stroke clinical tools for thromboembolic risk (-Table 1). Twenty-nine studies directly compared the predictive ability for thromboembolic events of the $\mathrm{CHADS}_{2}$ risk score with other risk scores, ${ }^{17-45} 24$ compared $\mathrm{CHA}_{2} \mathrm{DS}_{2}$ VASc, ${ }^{18-21,23,24,26,37,39-54} 6$ compared Framingham $^{18,24,33,34,37,55}$ and 4 compared ABC stroke. ${ }^{54,56-58} c$ Statistics for predicting thromboembolic risk, when available, are reported in - Supplementary Table $\mathbf{S 3}$ (available in the online version). Sufficient data existed to permit metaanalysis of studies evaluating $c$-statistics for the $\mathrm{CHADS}_{2}$ score using a continuous score ( - Fig. 2A) and categorical score (-Fig. 2B), the $\mathrm{CHA}_{2} \mathrm{DS}_{2}$-VASc continuous score (- Fig. 2C) and categorical score (-Fig. 2D), the Framingham categorical score (-Fig. 2E) and the ABC stroke categorical score (-Fig. 2F). For both the continuous and categorical $\mathrm{CHADS}_{2}$ scores (continuous: 14 studies with 489,335 patients; categorical: 16 studies, 548,464 patients; - Table 2), there was moderate strength of evidence that the scores provide limited prediction of stroke events (continuous: c-statistic of 0.69; 95\% CI, 0.66-0.73; categorical: $c$-statistic of 0.66 ; $95 \%$ $\mathrm{CI}, 0.63-0.69)$. There was also moderate strength of evidence (16 studies, 511,481 patients) that the continuous $\mathrm{CHA}_{2} \mathrm{DS}_{2}-$
VASc score provides limited prediction of stroke events ( $c$ statistic of $0.66 ; 95 \% \mathrm{CI}, 0.63-0.69)$. For the categorical $\mathrm{CHA}_{2} \mathrm{DS}_{2}$-VASc score (13 studies, 496,683 patients), there was low strength evidence of its ability to predict stroke risk (c-statistic of $0.64 ; 95 \% \mathrm{CI}, 0.58-0.70)$. Based on a metaanalysis of 6 studies (282,572 patients), we found moderate strength of evidence that the categorical Framingham score provides limited prediction of stroke events ( $c$-statistic of 0.63 ; 95\% CI, 0.62-0.65). For the categorical ABC score (4 studies, 25,614 patients), we found a moderate strength of evidence of limited prediction of stroke events ( $c$-statistic of 0.67; 95\% CI, 0.63-0.71) (-Table 2).

Seven imaging studies examined specific anatomical findings and their association with stroke risk in patients with $\mathrm{AF}^{59-65}$ Imaging studies included MRI, magnetic resonance angiography quantification of left atrial appendage dimensions, transoesophageal echocardiography and transthoracic echocardiography. There was insufficient evidence for the relationship between findings on echocardiography (transthoracic) and subsequent stroke based on 7 studies (4 low risk of bias, 3 medium risk of bias; 4,962 patients) that reported discrepant results.

We found 20 studies that evaluated either the predictive role of international normalized ratio (INR), pattern of AF, renal impairment or other risk factors. ${ }^{31,43,48,57,66-75}$ There was insufficient evidence, however, for further meta-analysis of the results. These abstracted data are in the full report.

\section{Predicting Bleeding Risk in Patients with AF}

Of the 38 studies which explored bleeding risk in patients with AF, 26 studies evaluated various risk scores (BRI, HEMORR $_{2}$ HAGES, HAS-BLED, ATRIA, ABC) for estimating the outcome of major bleeding risk in patients with $\mathrm{AF}$, including patients on warfarin, aspirin and no anti-thrombotic therapy. ${ }^{9,18,21,22,46,54,76-97}$ Thirteen studies (10 low risk of bias, 2 medium risk of bias, 1 high risk of bias; 351,985 patients) compared different risk scores (BRI, HEMORR $_{2}$ HAGES, HAS-BLED, ATRIA, ABC) in predicting major bleeding events in AF patients on warfarin. These studies differed markedly in population, major bleeding rates and statistics reported for evaluating risk prediction scores for major bleeding events.

Assessment of major bleeding events based on individual risk factors was reported by 17 studies (-Supplementary Table 54, available in the online version). Eight of these ( 7 low risk of bias, 1 medium risk of bias; 322,010 patients) evaluated the risk of major bleeding in patients with chronic kidney disease (CKD). All studies demonstrated increased risk of bleeding in patients with CKD (moderate strength of evidence). Other risk factors abstracted included the impact of INR, age, prior stroke, presence of heart disease, diabetes mellitus, sex, cancer, race/ethnicity and cognitive impairment; however, the evidence was insufficient to support findings (results in full report).

Most available studies for KQ2 included ICH within the outcome 'major bleeding', but three studies presented this outcome separately. One of these studies evaluated both HAS-BLED and HEMORR ${ }_{2}$ HAGES, $^{18}$ another study evaluated 


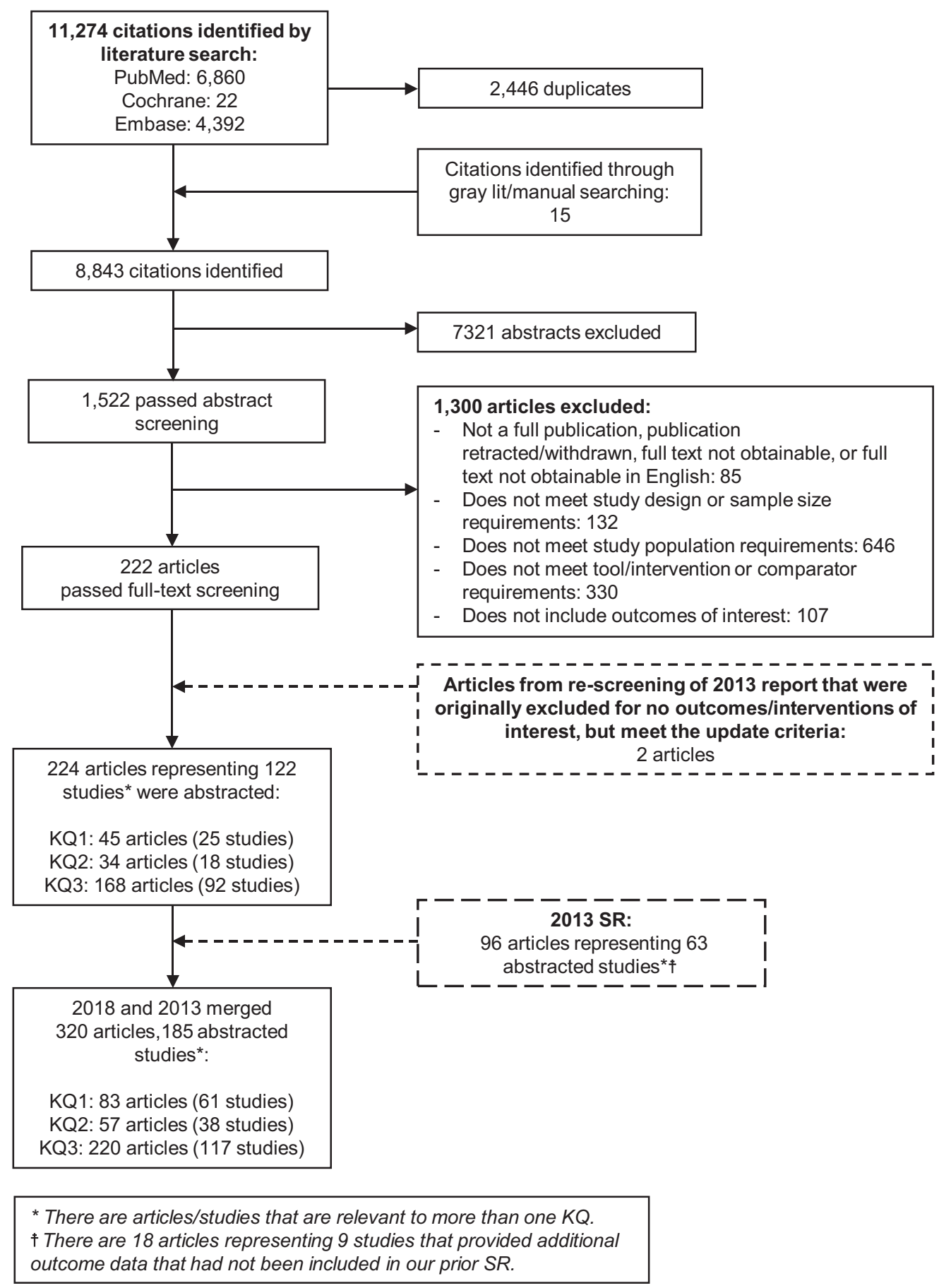

Fig. 1 Literature flow diagram. KQ, key question.

both HAS-BLED and ATRIA ${ }^{97}$ and a third study evaluated the INR. ${ }^{66}$ The single included study comparing HAS-BLED and HEMORR $_{2}$ HAGES did not show a statistically significant difference between the risk scores in prediction abilities for ICH in any patient population. Better understanding of ICH risk prediction will be particularly important, because this represents the most devastating variety of major bleeding event that patients on anticoagulation suffer.

The comparative risk discrimination abilities of each clinical tool was evaluated, when data were available, for (1) major bleeding risk in AF patients on warfarin, (2) AF patients on aspirin alone, (3) AF patients not on therapy and (4) ICH risk in AF patients on warfarin (see - Supplementary Table S5 for $c$-statistics, available in the online version). For AF patients on warfarin, evidence favoured HAS-BLED based on two studies demonstrating that it has significantly higher prediction (by c-statistic) for major bleeding events than other scores among patients on warfarin, but the majority of studies showed no statistically significant differences in prediction abilities, reducing the strength of evidence (moderate; - Table 3). For AF patients on aspirin alone, three studies ( 2 low risk of bias, 1 medium risk of bias; 177,538 
Table 1 Description and interpretation of included risk scores

\begin{tabular}{|c|c|c|c|}
\hline $\begin{array}{l}\text { Thromboembolic } \\
\text { risk score }\end{array}$ & Reference & Risk factors included & Interpretation \\
\hline $\mathrm{CHADS}_{2}$ & Gage et al, $2001^{35}$ & $\begin{array}{l}\text { Congestive heart failure, hyper- } \\
\text { tension, age } \geq 75 \text {, diabetes mel- } \\
\text { litus, prior stroke/transient } \\
\text { ischaemic attack [ } 2 \text { points] }\end{array}$ & $\begin{array}{l}\text { Low }(0) \text {, moderate }(1-2) \text {, high } \\
(3-6)\end{array}$ \\
\hline $\mathrm{CHA}_{2} \mathrm{DS}_{2}$-VASC & Lip et al, $2010^{37}$ & $\begin{array}{l}\text { Congestive heart failure/left } \\
\text { ventricular ejection fraction } \\
\leq 40 \% \text {, hypertension, age } \geq 75 \\
\text { [2 points], diabetes mellitus, } \\
\text { prior stroke/transient ischaemic } \\
\text { attack/thromboembolism } \\
{[2 \text { points], vascular disease, age }} \\
65-74, \text { sex category female }\end{array}$ & $\begin{array}{l}\text { Low }(0) \text {, moderate }(1) \text {, high (2- } \\
9)\end{array}$ \\
\hline Framingham & & $\begin{array}{l}\text { Advancing age, female sex, } \\
\text { increasing systolic blood pres- } \\
\text { sure, prior stroke or transient } \\
\text { ischaemic attack and diabetes }\end{array}$ & \\
\hline$A B C$ & Hijazi et al, $2016^{93}$ & $\begin{array}{l}\text { Age, biomarkers (cTnl-hs and NT- } \\
\text { proBNP), and clinical history } \\
\text { (prior stroke/TIA) }\end{array}$ & $\begin{array}{l}\text { Low }<1 \% \text {, moderate } 1-2 \% \text {, } \\
\text { high }>2 \%\end{array}$ \\
\hline Bleeding risk score & Reference & Risk factors included & Interpretation \\
\hline$A B C$ & Hijazi et al, $2016^{93}$ & $\begin{array}{l}\text { Age, biomarkers [GDF-15, cTnT- } \\
\text { hs, and haemoglobin], and clin- } \\
\text { ical history [previous bleeding] }\end{array}$ & $\begin{array}{l}\text { Low }<1 \% \text {, medium } 1-2 \% \text {, } \\
\text { high }>2 \%\end{array}$ \\
\hline ATRIA & Fang et al, $2011^{76}$ & $\begin{array}{l}\text { Anaemia, renal disease }(\mathrm{CrCl} \\
<30) \text { ( } 3 \text { points each); age } \geq 75 \\
(2 \text { points); any prior bleeding, } \\
\text { hypertension ( } 1 \text { point each) }\end{array}$ & $\begin{array}{l}\text { Low }(0-3), \text { moderate }(4), \text { high } \\
(5-10)\end{array}$ \\
\hline BRI & Beyth et al, $1998^{109}$ & $\begin{array}{l}\text { Age } \geq 65 \text {, Gl bleed in past } 2 \text { wk, } \\
\text { previous stroke, co-morbidities } \\
\text { (recent MI, haematocrit }<30 \% \text {, } \\
\text { diabetes, creatinine }>1.5 \text { ), with } \\
1 \text { point for presence of each } \\
\text { condition and } 0 \text { if absent }\end{array}$ & $\begin{array}{l}\text { Low }(0), \text { moderate }(1-2) \text {, high } \\
(3-4)\end{array}$ \\
\hline HAS-BLED & Pisters et al, $2010^{9}$ & $\begin{array}{l}\text { Hypertension, abnormal renal } \\
(\mathrm{CrCl}<50) \text { or liver function ( } 1 \\
\text { point each); stroke, bleeding } \\
\text { history or predisposition, labile } \\
\text { INR (TTR }<60 \% \text { ), age }>65 \text {, } \\
\text { drugs of interest/alcohol ( } 1 \text { point } \\
\text { each) }\end{array}$ & $\begin{array}{l}\text { Low }(0), \text { moderate }(1-2) \text {, high } \\
(\geq 3)\end{array}$ \\
\hline $\mathrm{HEMORR}_{2} \mathrm{HAGES}$ & Gage et al, $2006^{79}$ & $\begin{array}{l}\text { Liver/renal disease, ethanol } \\
\text { abuse, malignancy, age }>75 \text {, } \\
\text { low platelet count or function, } \\
\text { re-bleeding risk, uncontrolled } \\
\text { hypertension, anaemia, genetic } \\
\text { factors (CYP2C9), risk of fall or } \\
\text { stroke ( } 1 \text { point for each risk fac- } \\
\text { tor present with } 2 \text { points for } \\
\text { previous bleed) }\end{array}$ & $\begin{array}{l}\text { Low }(0-1) \text {, moderate }(2-3) \text {, high } \\
(\geq 4)\end{array}$ \\
\hline
\end{tabular}

Abbreviations: ABC, age, biomarkers, clinical history; ATRIA, Anticoagulation and Risk Factors in Atrial Fibrillation; BRI, Bleeding Risk Index; CrCl, creatinine clearance; CTnT-hs, high-sensitivity cardiac troponin T; GDF, growth differentiation factor-15; GI, gastrointestinal; HAS-BLED, Hypertension, Abnormal renal/liver function, Stroke, Bleeding history or predisposition, Labile international normalized ratio, Elderly (> 65 years), Drugs/ alcohol concomitantly; HEMORR 2 HAGES, Hepatic or renal disease, Ethanol abuse, Malignancy, Older (age $>75$ years), Reduced platelet count or function, Re-bleeding risk (2 points), Hypertension (uncontrolled), Anaemia, Genetic factors, Excessive fall risk, Stroke; INR, international normalized ratio; MI, myocardial infarction; TTR, time in therapeutic range. 


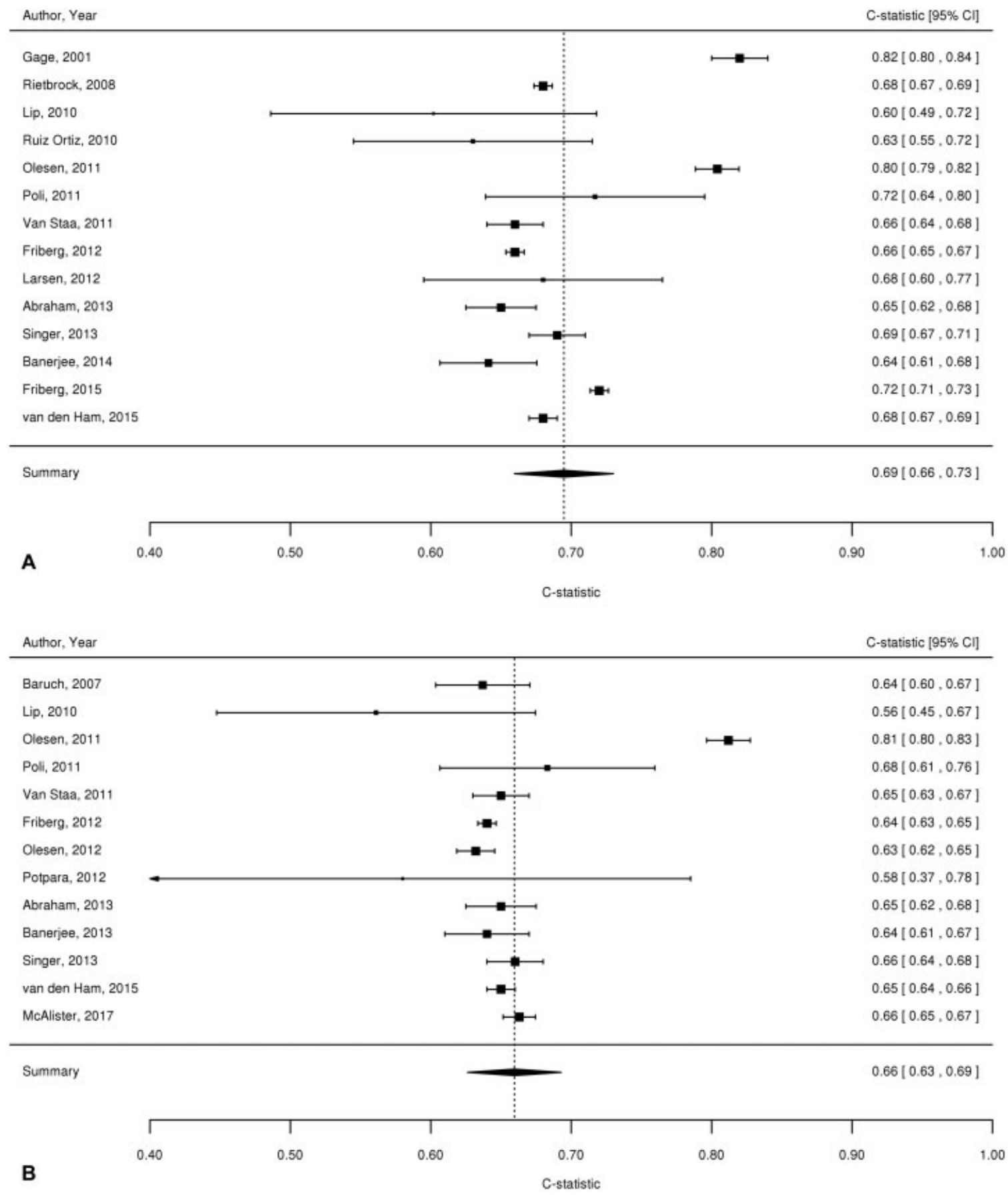

Fig. 2 (A-E) Summary estimate of $c$-statistics for prediction ability of clinical tools for thromboembolic risk (A) CHADS $_{2}$ continuous score. (B) $\mathrm{CHADS}_{2}$ categorical score. (C) $\mathrm{CHA}_{2} \mathrm{DS}_{2}$-VASc continuous score. (D) $\mathrm{CHA}_{2} \mathrm{DS}_{2}$-VASc categorical score. (E) Framingham categorical score. (F) $A B C$ stroke categorical score.

patients) comparing different combinations of bleeding risk scores (BRI, HEMORR ${ }_{2}$ HAGES and HAS-BLED) in predicting major bleeding events showed no statistically significant differences (low strength of evidence). Among AF patients not on therapy, six studies ( 4 low risk of bias, 2 medium risk of bias; 310,607 patients) comparing different combinations of bleeding risk scores (BRI, HEMORR ${ }_{2}$ HAGES, HAS-BLED and ATRIA) in predicting major bleeding events showed no statistically significant differences (low strength of evidence). Evaluating ICH in AF patients on warfarin, one study (low risk of bias; 48,599 patients) compared HEMOR$\mathrm{R}_{2}$ HAGES and HAS-BLED in predicting ICH. This study showed no statistically significant difference in prediction abilities between the two scores (low strength of evidence).

\section{Discussion}

Our review included studies comparing the diagnostic accuracy and impact on clinical decision-making of available clinical tools, imaging tools and associated risk factors for 


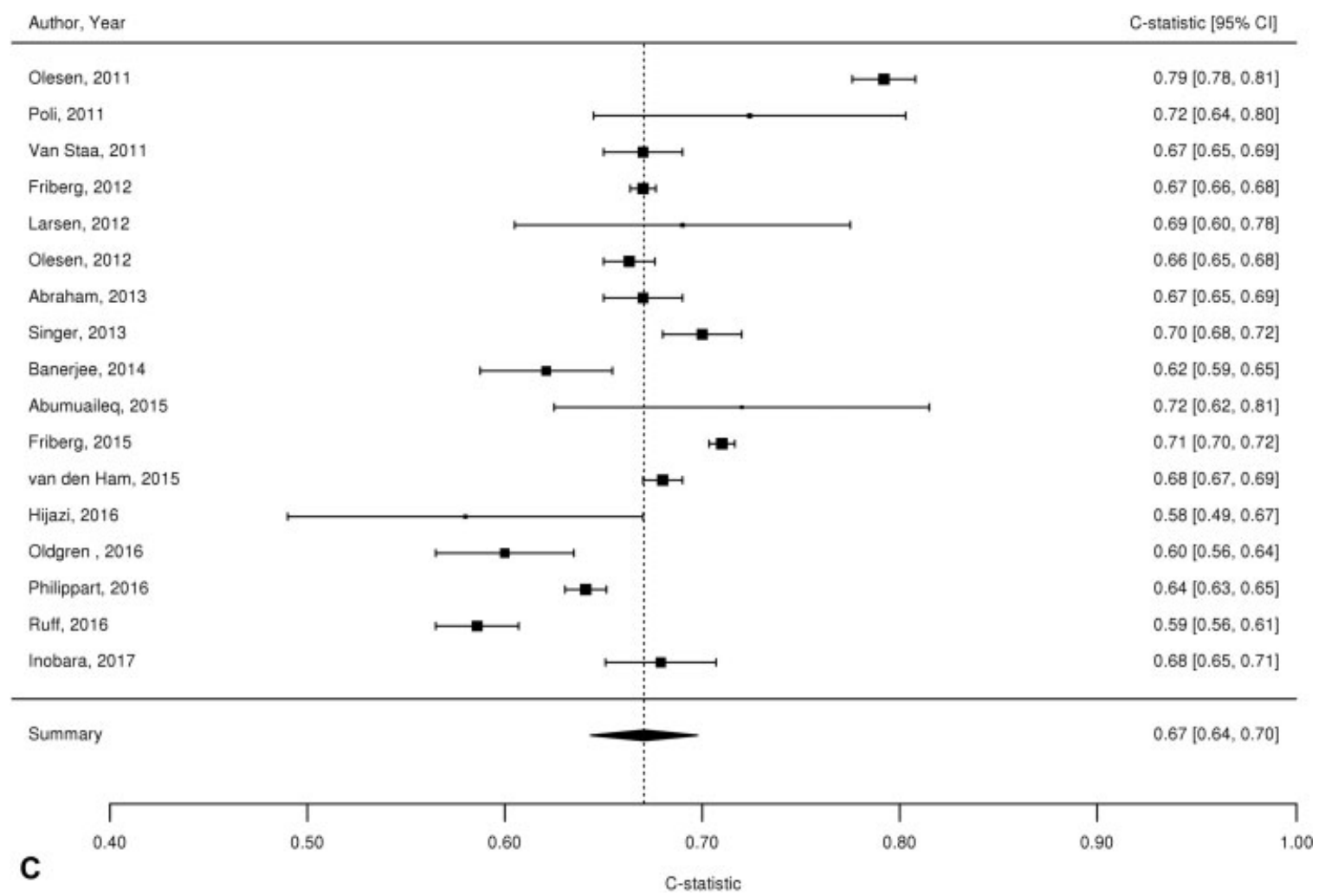

Fig. 2 (Continued)

predicting thromboembolic and bleeding risk in patients with AF. For predicting thromboembolic risk, the $\mathrm{CHADS}_{2}$, $\mathrm{CHA}_{2} \mathrm{DS}_{2}$-VASc and $\mathrm{ABC}$ scores appeared similar and had the best predictive abilities given the available evidence, but this advantage was not substantial on an absolute basis. Imaging risk tools, however, found conflicting results when the presence of a left atrial thrombus was assessed, and there was insufficient evidence to support conclusions regarding the predictive ability of the presence of a left atrial thrombus. Among the tools for predicting risk of major bleeding and $\mathrm{ICH}$, there was a suggestion that HAS-BLED is the best score for predicting major bleeds in patients on warfarin, although it only has modest prediction abilities. However, the majority of studies for other patient scenarios showed no statistically significant differences in predictive accuracy among tools.

\section{Findings in Relation to What is Already Known}

ESC guidelines recommend using the $\mathrm{CHA}_{2} \mathrm{DS}_{2}$ VASc score, and AHA guidelines recommend using the $\mathrm{CHADS}_{2}$ or $\mathrm{CHA}_{2} \mathrm{DS}_{2}$-VASc to categorize thromboembolic risk when making treatment decisions in patients with $\mathrm{AF} .{ }^{98}$ Additionally, recent American College of Clinical Pharmacy (ACCP), Australian and New Zealand (ANZ), and Asia Pacific Heart Rhythm Society (APHRS) guidelines endorse using the $\mathrm{CHA}_{2} \mathrm{DS}_{2}$-VASc score (excluding sex in the calculation under ACCP and ANZ guidelines) to identify low-risk patients that can be excluded from anticoagulation. ${ }^{99-101}$ In the current
CER, we found that of the available risk scores, the $\mathrm{CHADS}_{2}$ and $\mathrm{CHA}_{2} \mathrm{DS}_{2}$ VASc scores are the most commonly studied and that the $\mathrm{CHADS}_{2}, \mathrm{CHA}_{2} \mathrm{DS}_{2}$-VASc and $\mathrm{ABC}$ risk scores appeared to be similar and to have the highest predictive ability for stroke events. While some studies have explored the inclusion of biomarkers in stroke risk scores such as the $\mathrm{ABC}$ stroke risk score, and preliminary evidence supports the $\mathrm{ABC}$ score being comparable to $\mathrm{CHADS}_{2}$ and $\mathrm{CHA}_{2} \mathrm{DS}_{2}$-VASc, the experience with $\mathrm{ABC}$ is limited and more data are needed on the contribution of these and other biomarkers to the overall risk assessment. Further, few comparisons of the ABC score in predicting thromboembolic risk have been completed in 'real-world' populations, which may better clarify its predictive ability. ${ }^{102}$

In predicting bleeding risk, our review found limited evidence favouring the HAS-BLED risk score based on two studies demonstrating that it has a significantly higher predictive ability for major bleeding events than other scores among patients on warfarin. The majority of studies, however, showed no statistically significant differences in prediction, which reduced the strength of evidence. Recent evidence suggests that inclusion of time to therapeutic range (TTR), included in the HAS-BLED score, might enhance the predictive ability of other bleeding scores. ${ }^{54,87}$ Bleeding risk scores are not included in the most recent AHA/ACC guideline recommendations on $\mathrm{AF}$, and they are generally not used to decide whether to prescribe an oral anticoagulant to individual patients. However, bleeding risk scores may inform shared decision-making discussions of the risks of stroke and bleeding incorporating patients' values and 


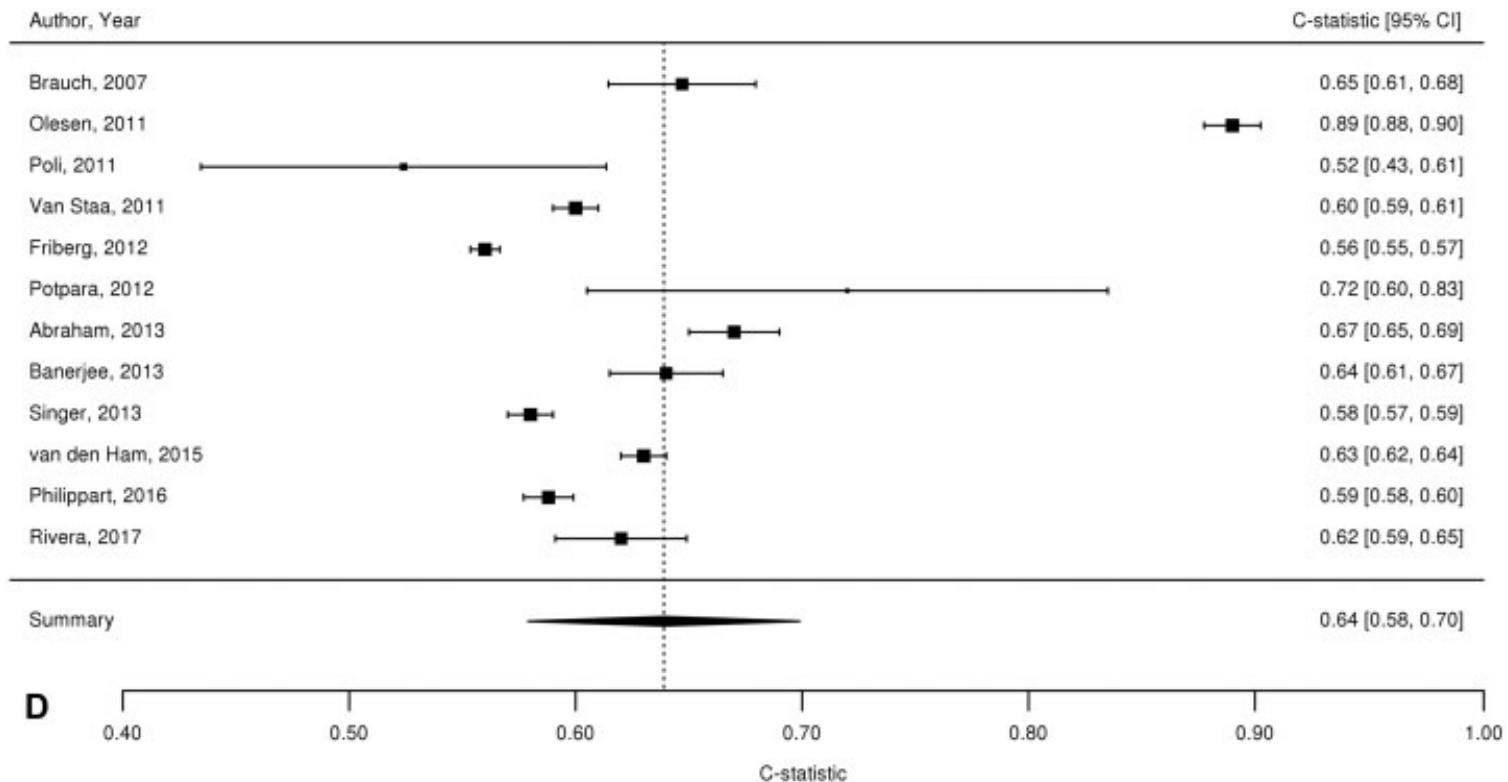

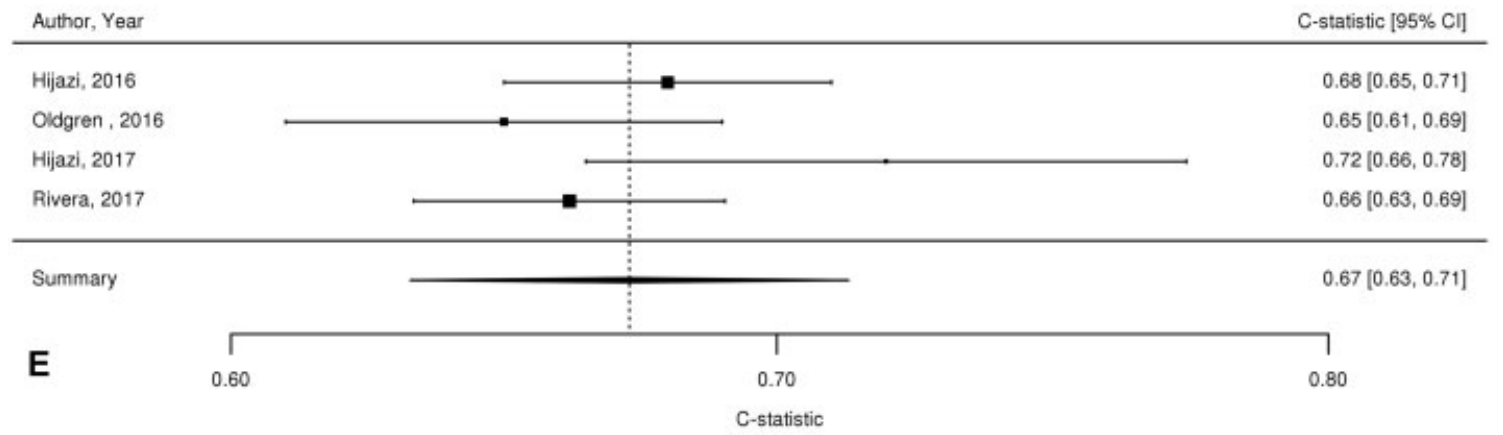

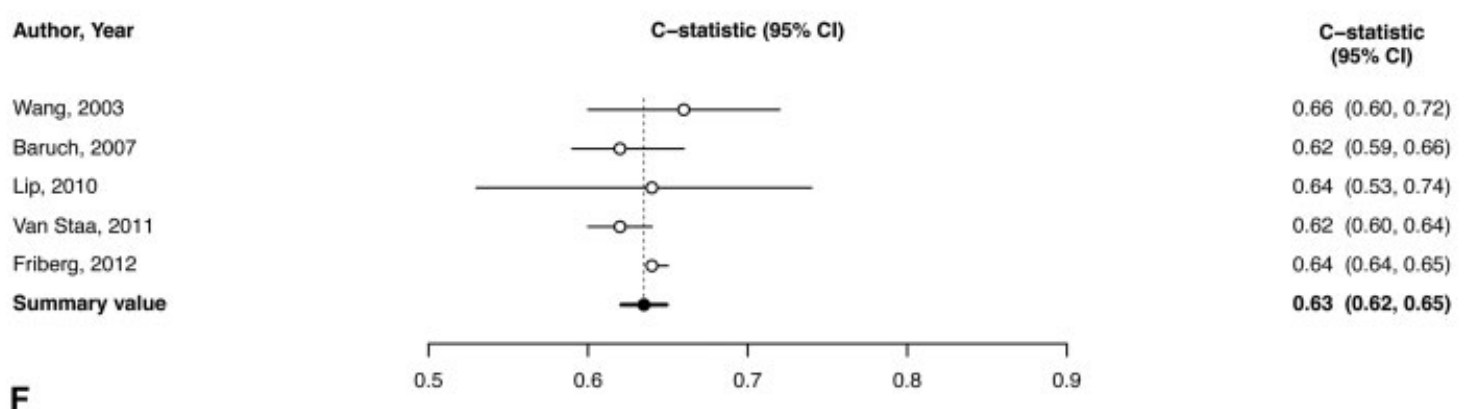

Fig. 2 (Continued)

preferences. As more data on stroke and bleeding risk scores emerge, it is possible that improvement in the tools and methods for risk stratification of both stroke and bleeding will be important to better individualize treatment using different oral anticoagulants in patients with AF.

\section{Limitations of the Evidence Base and the Comparative Effectiveness Review Process}

Comparisons across studies were difficult due to varying categorical arrangements of stroke risk scores, inter-study differences in approach to calculating some of the bleeding risk scores, limited comparison of bleeding risk scores across populations, heterogeneous patient populations and the variability in treating patients with anti-platelets and oral anticoagulants. It is known that risk scores correlate to differing event rates based on patient setting and treatment, such as whether they are in a clinical trial or in the outpatient setting, which further added to between study event rate discrepancies. ${ }^{103}$ Additionally, there was inconsistency among individual studies in reporting measures of calibration, strength of association and diagnostic accuracy. While the nature of a meta-analysis precludes the ability to directly account for individual study-level bias, we were able to 


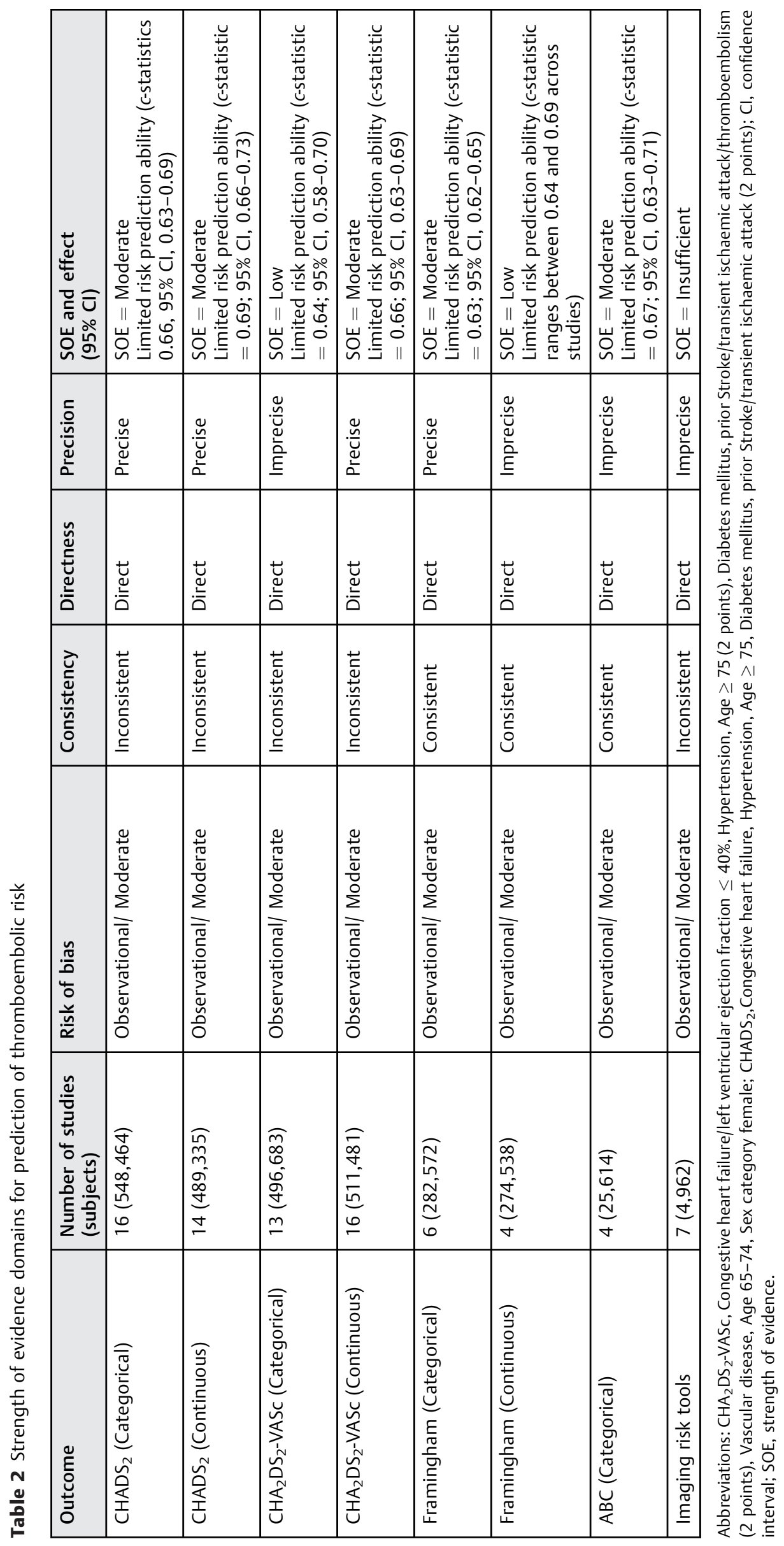




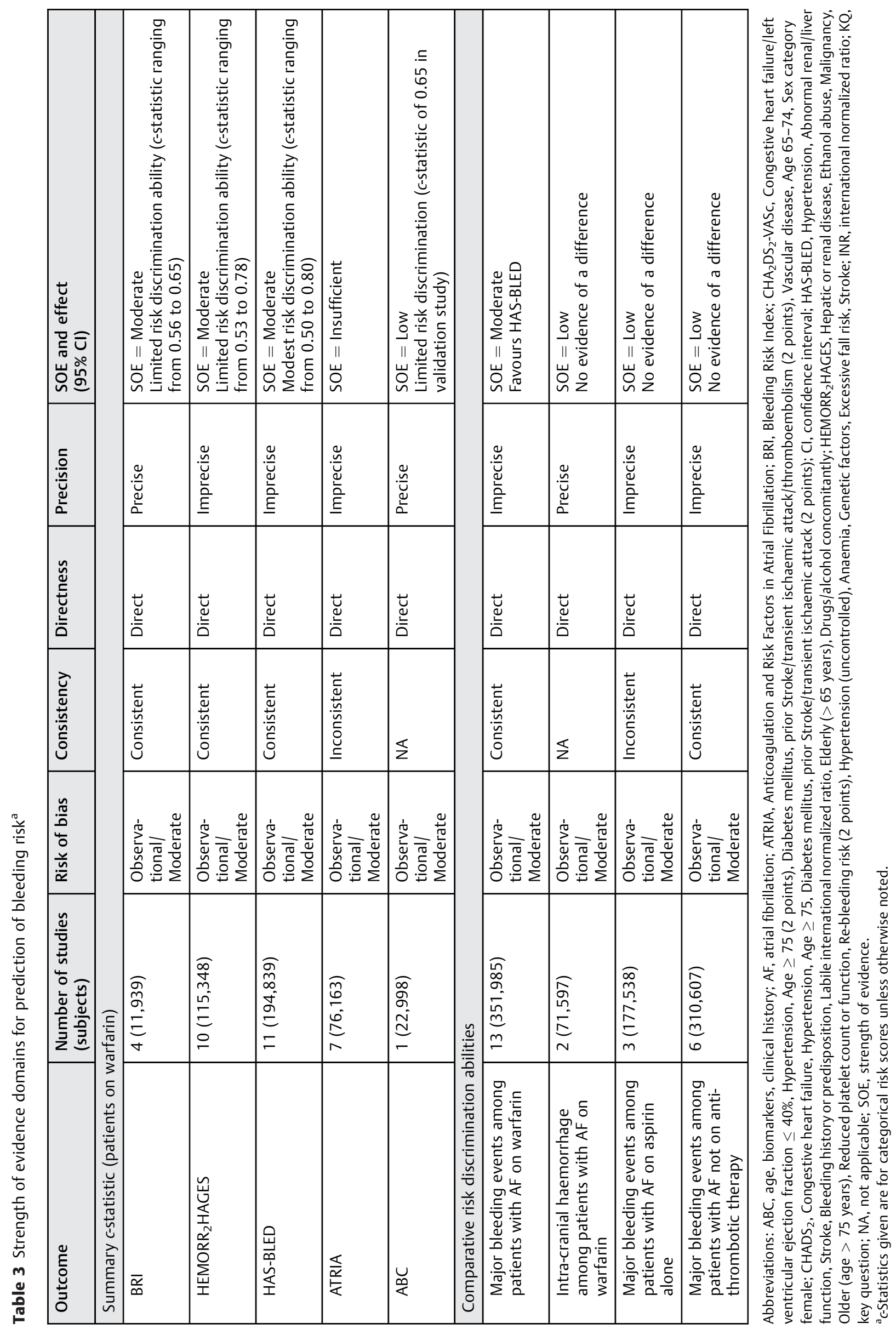


carefully assess for risk of bias, consistency, directness, precision and strength of evidence as outlined by best practice guidelines in systematic review methodology.

Further, our conclusions may be limited by the limitations in the development and validation of risk scores. Specifically, although many of the studies use clinical data sources to derive or validate these risk scores, some studies relied on billing data and institutional electronic medical records to identify patients with $\mathrm{AF}$ and co-morbidity information, which could under-estimate stroke risk due to lack of clinical adjudication of events. Likewise, lack of validated results or common event definitions for the endpoints of thromboembolism and bleeding could have under-estimated the performance of these risk scores. Additionally, lack of standard definitions for co-morbidities such as heart failure, diabetes mellitus and hypertension could also lead to discrepancies across studies validating the various risk scores. Moreover, our review included both ambulatory and hospitalized patients, which inherently introduces bias in comparing studies and results in heterogeneity with regards to stability of covariates, concomitant medications, stroke inducing procedures, etc.

Our review methods also had limitations. Our study was limited to English-language publications and excluded studies conducted exclusively in Asia, Africa or the Middle East. We also limited our analysis to studies published since 2000 as the recent literature was considered the most relevant to today's clinical and policy uncertainties. Lastly, we were unable to include systematic review of all available clinical risk score tools for stroke and bleeding risk. We are aware of other tools, such as QStroke and ORBIT scores, but our scope was focused on the scores used most frequently in clinical settings and prioritized through the stakeholder panel and topic refinement process with PCORI.

\section{Research Recommendations}

In our analyses, we have identified several areas for recommended future research. Given the aforementioned limitations of the currently available studies, further studies are needed that: (1) utilize complete data; (2) use validated clinical outcomes; and (3) compare all available risk scores using consistent and appropriate statistical evaluations.

Despite the availability and validation of numerous tools for both stroke and bleeding risk assessment in patients with non-valvular AF, meaningful comparisons of the tools could not be performed in this CER. Although the 2014 AHA/ACC guideline recommends using the $\mathrm{CHA}_{2} \mathrm{DS}_{2}$-VASc score for stroke risk stratification and that all patients with a $\mathrm{CHA}_{2} \mathrm{DS}_{2}$-VASc score of $\geq 2$ be considered for oral anticoagulant therapy, the guideline acknowledged the limitation of current risk tools, including the $\mathrm{CHA}_{2} \mathrm{DS}_{2}$-VASc score, to identify patients at high risk for thromboembolic risk. As a response to this poor predictive ability in high-risk patients, recently published ACCP, ANZ and APHRS guidelines suggest using the $\mathrm{CHA}_{2} \mathrm{DS}_{2}$-VASc score to identify low-risk patients in the initial step of determining whether anti-thrombotic therapy should be offered. ${ }^{99-101}$ Whether biomarkers such as brain natriuretic peptide, $\mathrm{C}$-reactive protein or troponin can enhance the $\mathrm{CHA}_{2} \mathrm{DS}_{2}$-VASc score and as a result be incorporated in guideline recommendations remains to be seen.

Also, the current ACC/AHA guidelines ${ }^{7}$ do not recommend use of bleeding risk scores, but rather focusing on modifiable bleeding risks. Our results found moderate strength of evidence for modest risk discrimination of the HAS-BLED score; how this modestly predictive score could potentially be utilized in clinical treatment decisions has yet to be investigated. Preliminary data in non-clinical trial populations show that biomarkers may not enhance risk scores' predictive ability of bleeding risk and further research is needed to conclusively determine whether biomarkers (e.g. brain natriuretic peptide, C-reactive protein or troponin) can enhance these scores. ${ }^{102,104}$

With the growing prevalence of digitized medical records, there is an opportunity to continue to evaluate and modify risk prediction tools to improve their accuracy in predicting stroke and bleeding risk, particularly with newer anticoagulants diffusing into clinical practice. These records might also facilitate research investigating risk as a non-static variable, observing changes in risk factors as predictive for stroke or bleeding events. ${ }^{105,106}$ Also, newer clinical markers (e.g. MRI to assess scar), co-morbidities (i.e. renal failure, etc.) and biomarkers should be tested and validated with or alongside current risk tools to improve their prediction of both stroke and bleeding risks. Additionally, more specific guidelines on how to use risk scores and apply necessary therapies, possibly in the form of physician decision-support tools, will be important for clinical decision-making. Efforts to create computer-based clinical decision-making supporting tools are on-going and may represent a way to better integrate clinical risk tools into practice. ${ }^{107,108}$ Preliminary evidence of such decision support systems is discussed within the full AHRQ report.

In addition, although we are able to identify patients at risk for stroke, many of these patients are also at a high risk for bleeding. Thus, there is a need for a score that could be used for decision-making about anti-thrombotic therapy in AF patients taking into account both thromboembolic and bleeding risks. Scores that identify only patients at risk for stroke or only those at risk for bleeding are not so helpful since the clinical factors in these scores are usually similar and treatments which reduce one or the other risk may increase the other for the same patient. Another challenge is that both stroke events and bleeding events are on a spectrum of severity and therefore predicting overall stroke might not align with outcomes that matter most to patients. For example, some strokes may have symptoms lasting $<24$ hours with complete resolution, whereas others can cause death. It may be good for future risk tools to account for differences in severity of outcomes. Another research need specific to bleeding risk is a prospective comparison of the standard deviation of transformed INR ( $\mathrm{SDT}_{\text {INR }}$ ) and TTR to establish which variable has better predictive accuracy for major bleeding including $\mathrm{ICH}$.

Additionally, even assuming an optimal risk prediction score can be identified, further work is needed to clarify how scores should be used prospectively in clinical practice. 
Clinical risk scores must take into account the balance between simplicity and practicality versus accurate prediction, especially in a high-capacity clinical environment. While clinical risk scores are necessarily reductionist and cannot feasibly consider all patient parameters, our results here show moderate predictive ability of risk scores that can be calculated relatively easily from patient history and demographics. Future research might explore this trade-off between ease of implementation and increasing the predictive value of clinical risk scores with more difficult-to-obtain parameters such as biomarkers.

\section{Conclusion}

Overall, we found that $\mathrm{CHADS}_{2}, \mathrm{CHA}_{2} \mathrm{DS}_{2}$-VASc and $\mathrm{ABC}$ stroke scores have the best prediction for stroke events in patients with AF among the risk scores we reviewed, whereas HAS-BLED provides the best prediction for bleeding risk. Imaging tools require further evidence in regard to their appropriate use in clinical decision-making. Additionally, simple clinical decision tools are needed that incorporate both stroke risk and bleeding risk to assist providers treating patients with AF. Additional work will be required to develop risk tools for patients to discriminate those individuals with $\mathrm{AF}$ where the bleeding risk may be high enough to warrant more intensive follow-up and monitoring. These tools could be embedded into electronic medical record systems for point-of-care decision-making, developed into applications for smartphones and tablets or be delivered via web-based interfaces. Additional evidence of the use of these stroke and bleeding risk scores (and clinical decision tools which balance these risks) among patients on therapy is also required.

\section{What is known about this topic?}

- The comparative diagnostic accuracy and impact on clinical decisionmaking of available clinical and imaging tools for predicting thromboembolic and bleeding risk in patients with atrial fibrillation (AF) are uncertain.

\section{What does this paper adds?}

- $\mathrm{CHADS}_{2}, \mathrm{CHA}_{2} \mathrm{DS}_{2}$-VASc, and ABC risk scores have the best evidence to support prediction of stroke events.

- HAS-BLED has the best evidence to support prediction of bleeding risk.

- Imaging tools for stroke prediction require further evidence.

\section{Funding}

This project was funded under Contract No. 290-201500004-I from the Agency for Healthcare Research and Quality (AHRQ), U.S. Department of Health and Human Services. The authors of this manuscript are responsible for its content. Statements in the manuscript should not be construed as endorsement by the Patient-Centered
Outcomes Research Institute (PCORI), AHRQ and the U.S. Department of Health and Human Services. AHRQ retains a license to display, reproduce and distribute the data and the report from which this manuscript was derived under the terms of the agency's contract with the author.

\section{Conflict of Interest}

None.

\section{Acknowledgements}

The authors thank Jamie Conklin, MSLIS, for help with the literature search and retrieval; Samantha E. Bowen, PhD, and Amanda J. McBroom, PhD, for assistance with project leadership; and Liz Wing, MA, for editorial assistance.

\section{References}

1 Mozaffarian D, Benjamin EJ, Go AS, et al; American Heart Association Statistics Committee and Stroke Statistics Subcommittee. Heart disease and stroke statistics-2015 update: a report from the American Heart Association. Circulation 2015;131(04): e29-e322

2 Go AS, Hylek EM, Phillips KA, et al. Prevalence of diagnosed atrial fibrillation in adults: national implications for rhythm management and stroke prevention: the AnTicoagulation and Risk Factors in Atrial Fibrillation (ATRIA) Study. JAMA 2001;285 (18):2370-2375

3 Furberg CD, Psaty BM, Manolio TA, Gardin JM, Smith VE, Rautaharju PM. Prevalence of atrial fibrillation in elderly subjects (the Cardiovascular Health Study). Am J Cardiol 1994;74(03): 236-241

4 Lee WC, Lamas GA, Balu S, Spalding J, Wang Q, Pashos CL. Direct treatment cost of atrial fibrillation in the elderly American population: a Medicare perspective. J Med Econ 2008;11(02): 281-298

5 Thrall G, Lane D, Carroll D, Lip GY. Quality of life in patients with atrial fibrillation: a systematic review. Am J Med 2006;119(05): 448.e1-448.e19

6 Stewart S, Hart CL, Hole DJ, McMurray JJ. A population-based study of the long-term risks associated with atrial fibrillation: 20 -year follow-up of the Renfrew/Paisley study. Am J Med 2002; 113(05):359-364

7 January CT, Wann LS, Alpert JS, et al; American College of Cardiology/American Heart Association Task Force on Practice Guidelines. 2014 AHA/ACC/HRS guideline for the management of patients with atrial fibrillation: a report of the American College of Cardiology/American Heart Association Task Force on Practice Guidelines and the Heart Rhythm Society. J Am Coll Cardiol 2014;64(21):e1-e76

8 Inoue $\mathrm{H}$, Nozawa T, Hirai T, et al. Accumulation of risk factors increases risk of thromboembolic events in patients with nonvalvular atrial fibrillation. Circ J 2006;70(06):651-656

9 Pisters R, Lane DA, Nieuwlaat R, de Vos CB, Crijns HJ, Lip GY. A novel user-friendly score (HAS-BLED) to assess 1-year risk of major bleeding in patients with atrial fibrillation: the Euro Heart Survey. Chest 2010;138(05):1093-1100

10 Lopes RD, Crowley MJ, Shah BR, et al. Stroke prevention in atrial fibrillation. AHRQ Comp Eff Rev 2013

11 Agency for Healthcare Research and Quality. Methods Guide for Effectiveness and Comparative Effectiveness Reviews. Rockville, MD: Agency for Healthcare Research and Quality. Available at: https://www.effectivehealthcare.ahrq.gov/topics/cer-methodsguide/overview. Accessed November 27, 2017

12 Agency for Healthcare Research and Quality. Methods Guide for Medical Test Reviews. Rockville, MD: Agency for Healthcare Research and Quality. Available at: https://www. 
effectivehealthcare.ahrq.gov/topics/methods-guidance-tests/ overview-2012/. Accessed November 27, 2017

13 Whiting PF, Rutjes AWS, Westwood ME, et al; QUADAS-2 Group. QUADAS-2: a revised tool for the Quality Assessment of Diagnostic Accuracy studies. Ann Intern Med 2011;155(08):529-536

14 DerSimonian R, Laird N. Meta-analysis in clinical trials. Control Clin Trials 1986;7(03):177-188

15 Ohman EM, Granger CB, Harrington RA, Lee KL. Risk stratification and therapeutic decision making in acute coronary syndromes. JAMA 2000;284(07):876-878

16 Owens DK, Lohr KN, Atkins D, et al. AHRQ series paper 5: grading the strength of a body of evidence when comparing medical interventions-agency for healthcare research and quality and the effective health-care program. J Clin Epidemiol 2010;63(05):513-523

17 Olesen JB, Fauchier L, Lane DA, Taillandier S, Lip GYH. Risk factors for stroke and thromboembolism in relation to age among patients with atrial fibrillation: the Loire Valley Atrial Fibrillation Project. Chest 2012;141(01):147-153

18 Friberg L, Rosenqvist M, Lip GY. Evaluation of risk stratification schemes for ischaemic stroke and bleeding in 182678 patients with atrial fibrillation: the Swedish Atrial Fibrillation cohort study. Eur Heart J 2012;33(12):1500-1510

19 Olesen JB, Lip GY, Lane DA, et al. Vascular disease and stroke risk in atrial fibrillation: a nationwide cohort study. Am J Med 2012; 125(08):826.e13-826.e23

20 Potpara TS, Polovina MM, Licina MM, Marinkovic JM, Prostran MS, Lip GY. Reliable identification of "truly low" thromboembolic risk in patients initially diagnosed with "lone" atrial fibrillation: the Belgrade atrial fibrillation study. Circ Arrhythm Electrophysiol 2012;5(02):319-326

21 Olesen JB, Lip GY, Lindhardsen J, et al. Risks of thromboembolism and bleeding with thromboprophylaxis in patients with atrial fibrillation: a net clinical benefit analysis using a 'real world' nationwide cohort study. Thromb Haemost 2011;106(04):739-749

22 Poli D, Testa S, Antonucci E, Grifoni E, Paoletti O, Lip GYH. Bleeding and stroke risk in a real-world prospective primary prevention cohort of patients with atrial fibrillation. Chest 2011; 140(04):918-924

23 Olesen JB, Lip GY, Hansen ML, et al. Validation of risk stratification schemes for predicting stroke and thromboembolism in patients with atrial fibrillation: nationwide cohort study. BMJ 2011;342:d124

24 Van Staa TP, Setakis E, Di Tanna GL, Lane DA, Lip GY. A comparison of risk stratification schemes for stroke in 79,884 atrial fibrillation patients in general practice. J Thromb Haemost 2011;9(01): 39-48

25 Ad N, Henry L, Schlauch K, Holmes SD, Hunt S. The CHADS score role in managing anticoagulation after surgical ablation for atrial fibrillation. Ann Thorac Surg 2010;90(04):1257-1262

26 Poli D, Lip GY, Antonucci E, Grifoni E, Lane D. Stroke risk stratification in a "real-world" elderly anticoagulated atrial fibrillation population. J Cardiovasc Electrophysiol 2011;22(01):25-30

27 Ruiz Ortiz M, Romo E, Mesa D, et al. Oral anticoagulation in nonvalvular atrial fibrillation in clinical practice: impact of CHADS(2) score on outcome. Cardiology 2010;115(03):200-204

28 Connolly SJ, Ezekowitz MD, Yusuf S, et al; RE-LY Steering Committee and Investigators. Dabigatran versus warfarin in patients with atrial fibrillation. N Engl J Med 2009;361(12):1139-1151

29 Crandall MA, Horne BD, Day JD, et al. Atrial fibrillation significantly increases total mortality and stroke risk beyond that conveyed by the CHADS2 risk factors. Pacing Clin Electrophysiol 2009;32(08):981-986

30 Poli D, Antonucci E, Grifoni E, Abbate R, Gensini GF, Prisco D. Stroke risk in atrial fibrillation patients on warfarin. Predictive ability of risk stratification schemes for primary and secondary prevention. Thromb Haemost 2009;101(02):367-372

31 Morgan CL, McEwan P, Tukiendorf A, Robinson PA, Clemens A, Plumb JM. Warfarin treatment in patients with atrial fibrillation: observing outcomes associated with varying levels of INR control. Thromb Res 2009;124(01):37-41

32 Rietbrock S, Heeley E, Plumb J, van Staa T. Chronic atrial fibrillation: incidence, prevalence, and prediction of stroke using the Congestive heart failure, Hypertension, Age $>75$, Diabetes mellitus, and prior Stroke or transient ischemic attack (CHADS2) risk stratification scheme. Am Heart J 2008;156(01):57-64

33 Fang MC, Go AS, Chang Y, Borowsky L, Pomernacki NK, Singer DE; ATRIA Study Group. Comparison of risk stratification schemes to predict thromboembolism in people with nonvalvular atrial fibrillation. J Am Coll Cardiol 2008;51(08):810-815

34 Baruch L, Gage BF, Horrow J, et al. Can patients at elevated risk of stroke treated with anticoagulants be further risk stratified? Stroke 2007;38(09):2459-2463

35 Gage BF, Waterman AD, Shannon W, Boechler M, Rich MW, Radford MJ. Validation of clinical classification schemes for predicting stroke: results from the National Registry of Atrial Fibrillation. JAMA 2001;285(22):2864-2870

36 Ruiz-Nodar JM, Marín F, Manzano-Fernández S, et al. An evaluation of the $\mathrm{CHADS}_{2}$ stroke risk score in patients with atrial fibrillation who undergo percutaneous coronary revascularization. Chest 2011;139(06):1402-1409

37 Lip GY, Nieuwlaat R, Pisters R, Lane DA, Crijns HJ. Refining clinical risk stratification for predicting stroke and thromboembolism in atrial fibrillation using a novel risk factor-based approach: the euro heart survey on atrial fibrillation. Chest 2010;137(02):263-272

38 Ruiz Ortiz M, Romo E, Mesa D, et al. Predicting embolic events in patients with nonvalvular atrial fibrillation: evaluation of the CHADS2 score in a Mediterranean population [in Spanish]. Rev Esp Cardiol 2008;61(01):29-35

39 Olesen JB, Torp-Pedersen C, Hansen ML, Lip GY. The value of the CHA2DS2-VASc score for refining stroke risk stratification in patients with atrial fibrillation with a CHADS2 score 0-1: a nationwide cohort study. Thromb Haemost 2012;107(06):1172-1179

40 Fanola CL, Giugliano RP, Ruff CT, et al. A novel risk prediction score in atrial fibrillation for a net clinical outcome from the ENGAGE AF-TIMI 48 randomized clinical trial. Eur Heart J 2017; 38(12):888-896

41 Abraham JM, Larson J, Chung MK, et al. Does CHA2DS2-VASc improve stroke risk stratification in postmenopausal women with atrial fibrillation? Am J Med 2013;126(12):1143.e1-1143.e8

42 Singer DE, Chang Y, Borowsky LH, et al. A new risk scheme to predict ischemic stroke and other thromboembolism in atrial fibrillation: the ATRIA study stroke risk score. J Am Heart Assoc 2013;2(03):e000250

43 Lip GY, Connolly S, Yusuf S, et al; ERROES Investigators. Modification of outcomes with aspirin or apixaban in relation to CHADS(2) and CHA(2)DS(2)-VASc scores in patients with atrial fibrillation: a secondary analysis of the AVERROES study. Circ Arrhythm Electrophysiol 2013;6(01):31-38

44 Larsen TB, Lip GY, Skjøth F, Due KM, Overvad K, Hvilsted Rasmussen L. Added predictive ability of the CHA2DS2VASc risk score for stroke and death in patients with atrial fibrillation: the prospective Danish Diet, Cancer, and Health cohort study. Circ Cardiovasc Qual Outcomes 2012;5(03):335-342

45 van den Ham HA, Klungel OH, Singer DE, Leufkens HG, van Staa TP. Comparative performance of ATRIA, CHADS2, and CHA2DS2VASc risk scores predicting stroke in patients with atrial fibrillation: results from a National Primary Care Database. J Am Coll Cardiol 2015;66(17):1851-1859

46 Ruiz-Nodar JM, Marín F, Roldán V, et al. Should we recommend oral anticoagulation therapy in patients with atrial fibrillation undergoing coronary artery stenting with a high HAS-BLED bleeding risk score? Circ Cardiovasc Interv 2012;5(04):459-466

47 Haas S, Ten Cate H, Accetta G, et al; GARFIELD-AF Investigators. Quality of vitamin K antagonist control and 1-year outcomes in patients with atrial fibrillation: a global perspective from the GARFIELD-AF Registry. PLoS One 2016;11(10):e0164076 
48 Ruff CT, Giugliano RP, Braunwald E, et al. Cardiovascular biomarker score and clinical outcomes in patients with atrial fibrillation: a subanalysis of the ENGAGE AF-TIMI 48 randomized clinical trial. JAMA Cardiol 2016;1(09):999-1006

49 Fauchier L, Clementy N, Bisson A, et al. Should atrial fibrillation patients with only 1 nongender-related CHA2DS2-VASc risk factor be anticoagulated? Stroke 2016;47(07):1831-1836

50 Apostolakis S, Guo Y, Lane DA, Buller H, Lip GY. Renal function and outcomes in anticoagulated patients with non-valvular atrial fibrillation: the AMADEUS trial. Eur Heart J 2013;34(46):3572-3579

51 Allan V, Banerjee A, Shah AD, et al. Net clinical benefit of warfarin in individuals with atrial fibrillation across stroke risk and across primary and secondary care. Heart 2017;103(03):210-218

52 Bonde AN, Lip GY, Kamper AL, et al. Net clinical benefit of antithrombotic therapy in patients with atrial fibrillation and chronic kidney disease: a nationwide observational cohort study. J Am Coll Cardiol 2014;64(23):2471-2482

53 Forslund T, Wettermark B, Wändell P, von Euler M, Hasselström J, Hjemdahl P. Risks for stroke and bleeding with warfarin or aspirin treatment in patients with atrial fibrillation at different CHA(2)DS(2)VASc scores: experience from the Stockholm region. Eur J Clin Pharmacol 2014;70(12):1477-1485

54 Rivera-Caravaca JM, Roldán V, Esteve-Pastor MA, et al. Importance of time in therapeutic range on bleeding risk prediction using clinical risk scores in patients with atrial fibrillation. Sci Rep 2017;7(01):12066

55 Wang TJ, Massaro JM, Levy D, et al. A risk score for predicting stroke or death in individuals with new-onset atrial fibrillation in the community: the Framingham Heart Study. JAMA 2003; 290(08):1049-1056

56 Hijazi Z, Lindbäck J, Alexander JH, et al; ARISTOTLE and STABILITY Investigators. The ABC (age, biomarkers, clinical history) stroke risk score: a biomarker-based risk score for predicting stroke in atrial fibrillation. Eur Heart J 2016;37(20):1582-1590

57 Granger CB, Alexander JH, McMurray JJ, et al; ARISTOTLE Committees and Investigators. Apixaban versus warfarin in patients with atrial fibrillation. N Engl J Med 2011;365(11):981-992

58 Oldgren J, Hijazi Z, Lindbäck J, et al; RE-LY and ARISTOTLE Investigators. Performance and validation of a novel biomarker-based stroke risk score for atrial fibrillation. Circulation 2016;134(22):1697-1707

59 Beinart R, Heist EK, Newell JB, Holmvang G, Ruskin JN, Mansour M. Left atrial appendage dimensions predict the risk of stroke/ TIA in patients with atrial fibrillation. J Cardiovasc Electrophysiol 2011;22(01):10-15

60 Nair CK, Holmberg MJ, Aronow WS, Shen X, Li H, Lakkireddy D. Thromboembolism in patients with atrial fibrillation with and without left atrial thrombus documented by transesophageal echocardiography. Am J Ther 2009;16(05):385-392

61 Stöllberger C, Chnupa P, Abzieher C, et al. Mortality and rate of stroke or embolism in atrial fibrillation during long-term followup in the embolism in left atrial thrombi (ELAT) study. Clin Cardiol 2004;27(01):40-46

62 Stoddard MF, Singh P, Dawn B, Longaker RA. Left atrial thrombus predicts transient ischemic attack in patients with atrial fibrillation. Am Heart J 2003;145(04):676-682

63 Thambidorai SK, Murray RD, Parakh K, et al; ACUTE investigators. Utility of transesophageal echocardiography in identification of thrombogenic milieu in patients with atrial fibrillation (an ACUTE ancillary study). Am J Cardiol 2005;96(07):935-941

64 Gupta DK, Giugliano RP, Ruff CT, et al; Effective Anticoagulation with Factor Xa Next Generation in AF-Thrombolysis in Myocardial Infarction 48 (ENGAGE AF-IMI 48) Echocardiographic Study Investigators. The prognostic significance of cardiac structure and function in atrial fibrillation: the ENGAGE AF-TIMI 48 echocardiographic substudy. J Am Soc Echocardiogr 2016;29(06):537-544

65 Yarmohammadi H, Klosterman T, Grewal G, et al. Efficacy of the $\mathrm{CHADS}_{2}$ scoring system to assess left atrial thrombogenic milieu risk before cardioversion of non-valvular atrial fibrillation. Am J Cardiol 2013;112(05):678-683

66 Hylek EM, Go AS, Chang Y, et al. Effect of intensity of oral anticoagulation on stroke severity and mortality in atrial fibrillation. N Engl J Med 2003;349(11):1019-1026

67 Lind M, Fahlén M, Kosiborod M, Eliasson B, Odén A. Variability of INR and its relationship with mortality, stroke, bleeding and hospitalisations in patients with atrial fibrillation. Thromb Res 2012;129(01):32-35

68 Link MS, Giugliano RP, Ruff CT, et al; ENGAGE AF-TIMI 48 Investigators. Stroke and mortality risk in patients with various patterns of atrial fibrillation: results from the ENGAGE AF-TIMI 48 trial (Effective Anticoagulation With Factor Xa Next Generation in Atrial Fibrillation-Thrombolysis in Myocardial Infarction 48). Circ Arrhythm Electrophysiol 2017;10(01):e004267

69 Connolly SJ, Eikelboom J, Joyner C, et al; AVERROES Steering Committee and Investigators. Apixaban in patients with atrial fibrillation. N Engl J Med 2011;364(09):806-817

70 Jun M, James MT, Ma Z, et al; Alberta Kidney Disease Network. Warfarin initiation, atrial fibrillation, and kidney function: comparative effectiveness and safety of warfarin in older adults with newly diagnosed atrial fibrillation. Am J Kidney Dis 2017;69 (06):734-743

71 Friberg L, Benson L, Lip GY. Balancing stroke and bleeding risks in patients with atrial fibrillation and renal failure: the Swedish Atrial Fibrillation Cohort study. Eur Heart J 2015;36(05): 297-306

72 Banerjee A, Fauchier L, Vourc'h P, et al. Renal impairment and ischemic stroke risk assessment in patients with atrial fibrillation: the Loire Valley Atrial Fibrillation Project. J Am Coll Cardiol 2013;61(20):2079-2087

73 Flaker GC, Pogue J, Yusuf S, et al; Atrial Fibrillation Clopidogrel Trial With Irbesartan for Prevention of Vascular Events (ACTIVE) Investigators. Cognitive function and anticoagulation control in patients with atrial fibrillation. Circ Cardiovasc Qual Outcomes 2010;3(03):277-283

74 Ashburner JM, Go AS, Chang Y, et al. Effect of diabetes and glycemic control on ischemic stroke risk in AF patients: ATRIA study. J Am Coll Cardiol 2016;67(03):239-247

75 McMurray JJ, Ezekowitz JA, Lewis BS, et al; ARISTOTLE Committees and Investigators. Left ventricular systolic dysfunction, heart failure, and the risk of stroke and systemic embolism in patients with atrial fibrillation: insights from the ARISTOTLE trial. Circ Heart Fail 2013;6(03):451-460

76 Fang MC, Go AS, Chang Y, et al. A new risk scheme to predict warfarin-associated hemorrhage: the ATRIA (Anticoagulation and Risk Factors in Atrial Fibrillation) study. J Am Coll Cardiol 2011;58(04):395-401

77 Lip GY, Frison L, Halperin JL, Lane DA. Comparative validation of a novel risk score for predicting bleeding risk in anticoagulated patients with atrial fibrillation: the HAS-BLED (Hypertension, Abnormal Renal/Liver Function, Stroke, Bleeding History or Predisposition, Labile INR, Elderly, Drugs/Alcohol Concomitantly) score. J Am Coll Cardiol 2011;57(02):173-180

78 Shireman TI, Mahnken JD, Howard PA, Kresowik TF, Hou Q Ellerbeck EF. Development of a contemporary bleeding risk model for elderly warfarin recipients. Chest 2006;130(05):1390-1396

79 Gage BF, Yan Y, Milligan PE, et al. Clinical classification schemes for predicting hemorrhage: results from the National Registry of Atrial Fibrillation (NRAF). Am Heart J 2006;151 (03):713-719

80 Aspinall SL, DeSanzo BE, Trilli LE, Good CB. Bleeding Risk Index in an anticoagulation clinic. Assessment by indication and implications for care. J Gen Intern Med 2005;20(11):1008-1013

81 Olesen JB, Lip GYH, Hansen PR, et al. Bleeding risk in 'real world' patients with atrial fibrillation: comparison of two established bleeding prediction schemes in a nationwide cohort. J Thromb Haemost 2011;9(08):1460-1467 
82 Apostolakis S, Lane DA, Guo Y, Buller H, Lip GY. Performance of the HEMORR(2)HAGES, ATRIA, and HAS-BLED bleeding riskprediction scores in patients with atrial fibrillation undergoing anticoagulation: the AMADEUS (evaluating the use of SR34006 compared to warfarin or acenocoumarol in patients with atrial fibrillation) study. J Am Coll Cardiol 2012;60(09):861-867

83 Gallego P, Roldán V, Torregrosa JM, et al. Relation of the HASBLED bleeding risk score to major bleeding, cardiovascular events, and mortality in anticoagulated patients with atrial fibrillation. Circ Arrhythm Electrophysiol 2012;5(02):312-318

84 Roldan V, Marin F, Fernandez H, et al. Predictive value of the HASBLED and ATRIA bleeding scores for the risk of serious bleeding in a 'real world' anticoagulated atrial fibrillation population. Chest 2013;143(01):179-184

85 Lip GY, Banerjee A, Lagrenade I, Lane DA, Taillandier S, Fauchier L. Assessing the risk of bleeding in patients with atrial fibrillation: the Loire Valley Atrial Fibrillation project. Circ Arrhythm Electrophysiol 2012;5(05):941-948

86 Barnes GD, Gu X, Haymart B, et al. The predictive ability of the CHADS2 and CHA2DS2-VASc scores for bleeding risk in atrial fibrillation: the MAQI(2) experience. Thromb Res 2014;134(02): 294-299

87 Proietti M, Senoo K, Lane DA, Lip GY. Major bleeding in patients with non-valvular atrial fibrillation: impact of time in therapeutic range on contemporary bleeding risk scores. Sci Rep 2016; 6:24376

88 Jaspers Focks J, van Vugt SP, Albers-Akkers MT, et al. Low performance of bleeding risk models in the very elderly with atrial fibrillation using vitamin $\mathrm{K}$ antagonists. J Thromb Haemost 2016;14(09):1715-1724

89 Apostolakis S, Lane DA, Buller H, Lip GY. Comparison of the CHADS2, CHA2DS2-VASc and HAS-BLED scores for the prediction of clinically relevant bleeding in anticoagulated patients with atrial fibrillation: the AMADEUS trial. Thromb Haemost 2013;110(05):1074-1079

90 O'Brien EC, Simon DN, Thomas LE, et al. The ORBIT bleeding score: a simple bedside score to assess bleeding risk in atrial fibrillation. Eur Heart J 2015;36(46):3258-3264

91 Senoo K, Proietti M, Lane DA, Lip GY. Evaluation of the HAS-BLED, ATRIA, and ORBIT bleeding risk scores in patients with atrial fibrillation taking warfarin. Am J Med 2016;129(06):600-607

92 Esteve-Pastor MA, García-Fernández A, Macías M, et al; FANTASIIA Investigators. Is the ORBIT bleeding risk score superior to the HAS-BLED score in anticoagulated atrial fibrillation patients? Circ J 2016;80(10):2102-2108

93 Hijazi Z, Oldgren J, Lindbäck J, et al; ARISTOTLE and RE-LY Investigators. The novel biomarker-based $\mathrm{ABC}$ (age, biomarkers, clinical history)-bleeding risk score for patients with atrial fibrillation: a derivation and validation study. Lancet 2016;387 (10035):2302-2311

94 Lip GYH, Skjøth F, Nielsen PB, Kjældgaard JN, Larsen TB. The HASBLED, ATRIA, and ORBIT bleeding scores in atrial fibrillation patients using non-vitamin K antagonist oral anticoagulants. Am J Med 2018;131(05):574.e13-574.e27

95 Inohara T, Shrader P, Pieper K, et al. Association of atrial fibrillation clinical phenotypes with treatment patterns and outcomes: a multicenter registry study. JAMA Cardiol 2018;3(01):54-63

96 Proietti M, Hijazi Z, Andersson U, et al; RE-LY Investigators. Comparison of bleeding risk scores in patients with atrial fibrillation: insights from the RE-LY trial. J Intern Med 2018; 283(03):282-292

97 Yao X, Gersh BJ, Sangaralingham LR, et al. Comparison of the $\mathrm{CHA}_{2} \mathrm{DS}_{2}$-VASc, $\mathrm{CHADS}_{2}$, HAS-BLED, ORBIT, and ATRIA risk scores in predicting non-vitamin $\mathrm{K}$ antagonist oral anticoagulants-associated bleeding in patients with atrial fibrillation. Am J Cardiol 2017;120(09):1549-1556

98 Fuster V, Rydén LE, Cannom DS, et al; American College of Cardiology/American Heart Association Task Force on Practice
Guidelines; European Society of Cardiology Committee for Practice Guidelines; European Heart Rhythm Association; Heart Rhythm Society. ACC/AHA/ESC 2006 Guidelines for the Management of Patients with Atrial Fibrillation: a report of the American College of Cardiology/American Heart Association Task Force on Practice Guidelines and the European Society of Cardiology Committee for Practice Guidelines (Writing Committee to Revise the 2001 Guidelines for the Management of Patients With Atrial Fibrillation): developed in collaboration with the European Heart Rhythm Association and the Heart Rhythm Society. Circulation 2006;114(07):e257-e354

99 Brieger D, Amerena J, Attia J, et al; NHFA CSANZ Atrial Fibrillation Guideline Working Group. National Heart Foundation of Australia and the Cardiac Society of Australia and New Zealand: Australian Clinical Guidelines for the Diagnosis and Management of Atrial Fibrillation 2018. Heart Lung Circ 2018;27(10): 1209-1266

100 Lip GYH, Banerjee A, Boriani G, et al. Antithrombotic therapy for atrial fibrillation: CHEST guideline and expert panel report. Chest 2018:S0012-3692(18)32244-X

101 Chiang CE, Okumura K, Zhang S, et al. 2017 consensus of the Asia Pacific Heart Rhythm Society on stroke prevention in atrial fibrillation. J Arrhythm 2017;33(04):345-367

102 Rivera-Caravaca JM, Roldán V, Esteve-Pastor MA, et al. Long-term stroke risk prediction in patients with atrial fibrillation: comparison of the ABC-stroke and $\mathrm{CHA}_{2} \mathrm{DS}_{2}$-VASc scores. J Am Heart Assoc 2017;6(07):e006490

103 Rivera-Caravaca JM, Esteve-Pastor MA, Marín F, et al. A propensity score matched comparison of clinical outcomes in atrial fibrillation patients taking vitamin Kantagonists: comparing the "real-world" vs clinical trials. Mayo Clin Proc 2018;93(08):1065-1073

104 Proietti M, Rivera-Caravaca JM, Esteve-Pastor MA, Romiti GF, Marin F, Lip Gregory YH. Predicting bleeding events in anticoagulated patients with atrial fibrillation: a comparison between the HAS-BLED and GARFIELD-AF bleeding scores. JAHA 2018;7 (18):[Online at https://doi.org/10.1161/JAHA.1118.009766]

105 Chao TF, Lip GYH, Lin YJ, et al. Incident risk factors and major bleeding in patients with atrial fibrillation treated with oral anticoagulants: a comparison of baseline, follow-up and delta HAS-BLED scores with an approach focused on modifiable bleeding risk factors. Thromb Haemost 2018;118(04):768-777

106 Chao TF, Lip GYH, Liu CJ, et al. Relationship of aging and incident comorbidities to stroke risk in patients with atrial fibrillation. J Am Coll Cardiol 2018;71(02):122-132

107 Esteve-Pastor MA, Marín F, Bertomeu-Martinez V, et al; FANTASIIA Study Investigators. Do physicians correctly calculate thromboembolic risk scores? A comparison of concordance between manual and computer-based calculation of CHADS2 and CHA2 DS2 -VASc scores. Intern Med J 2016;46(05):583-589

108 Silbernagel G, Spirk D, Hager A, Baumgartner I, Kucher N. Electronic alert system for improving stroke prevention among hospitalized oral-anticoagulation-naïve patients with atrial fibrillation: a randomized trial. J Am Heart Assoc 2016;5(07):e003776

109 Beyth RJ, Quinn LM, Landefeld CS. Prospective evaluation of an index for predicting the risk of major bleeding in outpatients treated with warfarin. Am J Med 1998;105(02):91-99

110 Dechartres A, Trinquart L, Boutron I, Ravaud P. Influence of trial sample size on treatment effect estimates: meta-epidemiological study. BMJ 2013;346:f2304

111 Dechartres A, Altman DG, Trinquart L, Boutron I, Ravaud P. Association between analytic strategy and estimates of treatment outcomes in meta-analyses. JAMA 2014;312(06): 623-630

112 Abumuaileq RR, Abu-Assi E, López-López A, et al. Comparison between CHA2DS2-VASc and the new R2CHADS2 and ATRIA scores at predicting thromboembolic event in non-anticoagulated and anticoagulated patients with non-valvular atrial fibrillation. BMC Cardiovasc Disord 2015;15:156 
113 Banerjee A, Fauchier L, Bernard-Brunet A, Clementy N, Lip GY. Composite risk scores and composite endpoints in the risk prediction of outcomes in anticoagulated patients with atrial fibrillation. The Loire Valley Atrial Fibrillation Project. Thromb Haemost 2014;111(03):549-556

114 Hijazi Z, Lindahl B, Oldgren J, et al. Repeated measurements of cardiac biomarkers in atrial fibrillation and validation of the $A B C$ stroke score over time. J Am Heart Assoc 2017;6(06):e004851

115 McAlister FA, Wiebe N, Jun M, et al. Are existing risk scores for nonvalvular atrial fibrillation useful for prediction or risk adjustment in patients with chronic kidney disease? Can J Cardiol 2017;33(02):243-252

116 Philippart R, Brunet-Bernard A, Clementy N, et al. Oral anticoagulation, stroke and thromboembolism in patients with atrial fibrillation and valve bioprosthesis. The Loire Valley Atrial Fibrillation Project. Thromb Haemost 2016;115(05): 1056-1063

117 Bassand JP, Accetta G, Al Mahmeed W, et al; GARFIELD-AF Investigators. Risk factors for death, stroke, and bleeding in 28,628 patients from the GARFIELD-AF registry: Rationale for comprehensive management of atrial fibrillation. PLoS One 2018;13(01):e0191592

118 Sherwood MW, Nessel CC, Hellkamp AS, et al. Gastrointestinal bleeding in patients with atrial fibrillation treated with rivaroxaban or warfarin: ROCKET AF trial. J Am Coll Cardiol 2015;66 (21):2271-2281

119 Orkaby AR, Ozonoff A, Reisman JI, Miller DR, Zhao S, Rose AJ. Continued use of warfarin in veterans with atrial fibrillation after dementia diagnosis. J Am Geriatr Soc 2017;65(02):249-256
120 An J, Niu F, Zheng C, et al. Warfarin management and outcomes in patients with nonvalvular atrial fibrillation within an integrated health care system. J Manag Care Spec Pharm 2017;23(06):700-712

121 Phelps E, Delate T, Witt DM, Shaw PB, McCool KH, Clark NP. Effect of increased time in the therapeutic range on atrial fibrillation outcomes within a centralized anticoagulation service. Thromb Res 2018;163:54-59

122 Rivera-Caravaca JM, Roldán V, Esteve-Pastor MA, et al. Reduced time in therapeutic range and higher mortality in atrial fibrillation patients taking acenocoumarol. Clin Ther 2018;40(01):114-122

123 Goodman SG, Wojdyla DM, Piccini JP, et al; ROCKET AF Investigators. Factors associated with major bleeding events: insights from the ROCKET AF trial (rivaroxaban once-daily oral direct factor Xa inhibition compared with vitamin $\mathrm{K}$ antagonism for prevention of stroke and embolism trial in atrial fibrillation). J Am Coll Cardiol 2014;63(09):891-900

124 Hankey GJ, Stevens SR, Piccini JP, et al; ROCKET AF Steering Committee and Investigators. Intracranial hemorrhage among patients with atrial fibrillation anticoagulated with warfarin or rivaroxaban: the rivaroxaban once daily, oral, direct factor Xa inhibition compared with vitamin $\mathrm{K}$ antagonism for prevention of stroke and embolism trial in atrial fibrillation. Stroke 2014;45 (05):1304-1312

125 Renoux C, Coulombe J, Suissa S. Revisiting sex differences in outcomes in non-valvular atrial fibrillation: a population-based cohort study. Eur Heart J 2017;38(19):1473-1479

126 Hilkens NA, Algra A, Greving JP. Predicting major bleeding in ischemic stroke patients with atrial fibrillation. Stroke 2017;48 (11):3142-3144 